\title{
Zimt-Liquids schwächen pulmonale Immunabwehr
}

\author{
E-Zigaretten sind wahrscheinlich weniger gesundheitsschädlich als gewöhnliche Zigaretten, harmlos sind \\ sie deshalb nicht. Neue Forschungen zeigen, dass aromatisierte Liquids die lokale Immunabwehr in der \\ Lunge herabsetzen. Die Ergebnisse wurden auf dem ATS-Kongress in San Francisco vorgestellt.
}

Im Prinzip enthält der Rauch von E-Zigaretten die gleichen Toxine, Karzinogene und Partikel wie der von Tabakzigaretten, aber in deutlich geringerer Konzentration, erklärte Prof. Ilona Jaspers von der University of North Carolina in Chapel Hill. Die Trägerstoffe Propylenglykol und Glyzerin gelten als harmlos, auch wenn ein irritierendes Potenzial bei Inhalation letztlich nicht ausgeschlossen werden kann.

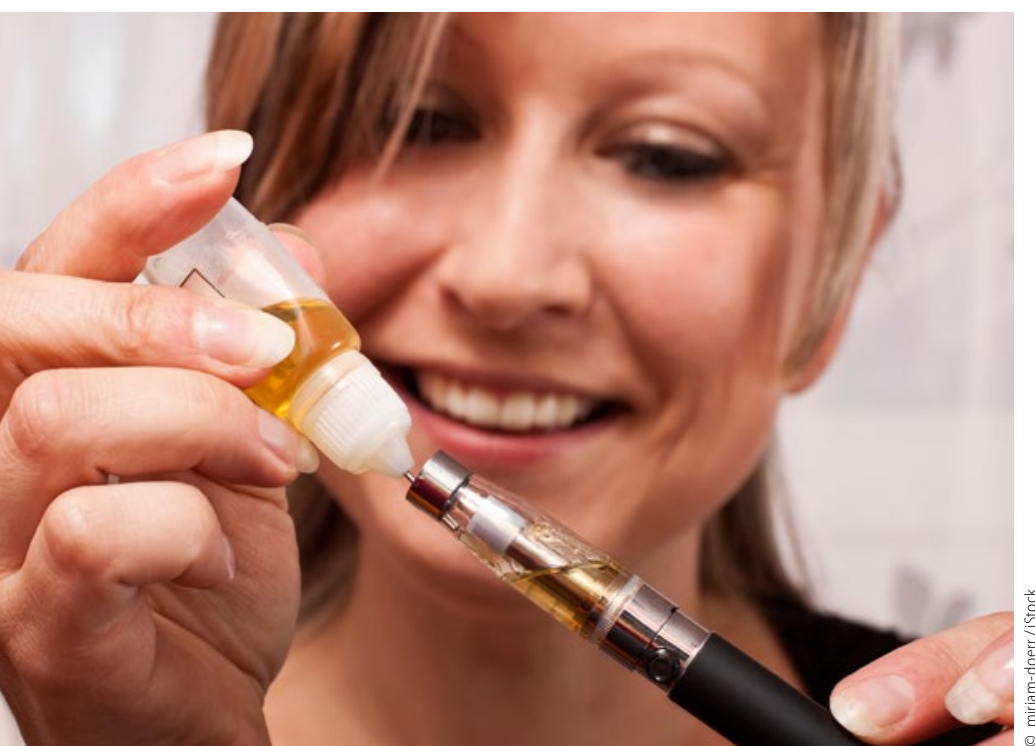

Sie duften zwar gut, allerdings beeinträchtigen Aromastoffe in den e-Zigaretten-Liquids die Effektivität von Immunzellen.

\section{Mangelnde Qualitätskontrolle}

Mehr Sorgen bereiten Jaspers die mangelnde Qualitätskontrolle und die nahezu unüberschaubare Vielzahl an Aromastoffen in den Liquids. Viele davon stehen auf der sogenannten GRASListe („Generally Regarded As Safe“) der FDA und werden deshalb als sicher angepriesen, untersucht sind sie aber nur für die orale Aufnahme. „Ob sie bei Inhalation auch so harmlos sind, ist völlig unklar“, betonte Jaspers beim ATS-Kongress 2016.

Ihr Team hat sich für die aktuelle Arbeit Aromastoffe auf Basis von Trans-Cinnamaldehyd näher angeschaut. Dieser Stoff verleiht Zimt den typischen Geruch und Geschmack. Die Katastrophenschutzbehörde FEMA stuft ihn als potenziell gefährlich bei Inhalation ein. Zimt-Liquids zählen dennoch derzeit zu den Bestsellern in den USA.

\section{Geringere Phagozytose-Kapazität}

Getestet wurden in vitro die Effekte auf Zellen des angeborenen Immunsystems, namentlich Makrophagen und neutrophile Granulozyten. Mit Cinnamaldehyd aromatisierte Liquids üben konzentrationsabhängige Effekte auf diese Zellen aus, berichtete Jaspers.

Unter dem am stärksten konzentrierten Liquid, einem Produkt namens Sini-cide, sank die Phagozytose-Kapazität der Makrophagen auf nur ein Drittel des Normalwerts. Neutrophile reagierten noch stärker auf die Liquids. Auch ihre PhagozytoseLeistung ging drastisch zurück. Außerdem stellten sie das für die Infektabwehr wichtige Trapping fast völlig ein.

Manuela Arand

\section{Schlafbezogene Atemstörungen}

\section{Schlechte Prognose bei Herzinsuffizienz plus isolierter zentraler Schlafapnoe}

\begin{abstract}
Patienten mit chronischer Herzinsuffizienz und isolierter zentraler Schlafapnoe haben eine schlechtere Prognose als herzschwache Patienten, die sowohl eine zentrale als auch eine obstruktive Schlafapnoe aufweisen.
\end{abstract}

Vor allem bei schwerer Herzinsuffizienz und zentraler Apnoe ist die Prognose für die betroffenen Patienten düster. Dann hilft auch keine adaptive Servo-Ventilation (ASV) mehr, berichteten Autoren um Renaud Tamisier aus Grenoble beim ATS-Meeting 2016.

Die Befunde sind vorläufig und stammen aus der FACE-Studie, einer nationalen Kohortenstudie aus Frankreich mit 301 herzinsuffizienten Patienten, von denen $70 \%$ eine zentrale und $30 \%$ eine gemischte Schlafapnoe aufwiesen. Bei $75 \%$ der Patienten war die Atemstörung ausgeprägt (AHI >30/h). Die Patienten mit nur zentraler Apnoe waren älter, dicker, litten häufi- ger an COPD und an schwereren Apnoen. Zentrale Schlafapnoen machen etwa 10 bis $20 \%$ aller Schlafapnoen aus. Bei Herzinsuffizienz haben sie mit $30 \%$ einen höheren Anteil. Die Patienten leiden an einem instabilen Schlaf, charakterisiert durch Phasen der Hyperventilation (bedingt durch $\mathrm{CO}_{2}$-Hypersensivität) und Hypoventilation.

Fazit der Autoren: Patienten mit Herzinsuffizienz und gemischter Apnoe könnten unter ASV eine bessere Prognose haben als solche mit nur zentraler Apnoe. Die Studie läuft weiter. Dr. med. Dirk Einecke 